\title{
Abordaje de la violencia laboral contra las mujeres en la justicia ordinaria laboral argentina*
}

\author{
The Workplace Violence Against Women in the Judgments \\ of the Argentinian Ordinary Labor Justice \\ Abordagem da violência laboral contra as mulheres \\ na justiça ordinária laboral argentina
}

JULIETA LOBATO ${ }^{\star *}$

FECHA DE RECEPCIÓN: 7 DE FEBRERO DE 2020. FeCHA DE APROBACIÓN: 17 DE ABRIL DE 2020

Doi: https://doi.org/10.12804/revistas.urosario.edu.co/sociojuridicos/a.8684

Para citar este artículo: Lobato, J. (2021). Abordaje de la violencia laboral contra las mujeres en la justicia ordinaria laboral argentina. Revista de Estudios Socio-Jurídicos, 23(1), 295-317. https://doi.org/10.12804/revistas.urosario.edu. co/sociojuridicos/a. 8684

\section{RESUMEN}

Este trabajo explora los estándares que utiliza la jurisprudencia en casos de violencia laboral contra las mujeres. La tesis principal sostiene que la justicia laboral reproduce dinámicas de dominación sexista, al no adecuar los parámetros de abordaje judicial a los estándares internacionales vigentes en materia de protección de los derechos de las mujeres. Para ello se analizará una muestra de jurisprudencia de la Cámara Nacional de Apelaciones del Trabajo, en la que se atenderá a dos cuestiones principales: las concepciones de violencia contra las mujeres en los ámbitos del trabajo y la configuración del cuadro probatorio y distribución de las cargas de prueba. Luego, se contrastarán estos

* Este trabajo se vio enriquecido por los diálogos y discusiones mantenidas con Leticia Vita, Martín Aldao, Laura Clérico y las/os participantes del grupo Género, Mercado de Trabajo y Cuidado del $14^{\circ}$ Congreso Nacional de Estudios del Trabajo (Buenos Aires, agosto 2019) coordinado por Laura Pautassi y María Eugenia Miguez. A todas/os ellas/os mi agradecimiento por sus observaciones y críticas. El trabajo es parte de una línea de investigación que pertenece al proyecto PICT 2015-3239 (Raíces): "Los DESC como Derechos Exigibles en Argentina: Aportes para una metodología de argumentación desde una perspectiva integral", cuyas investigadoras responsables son Leticia Vita y Laura Clérico. Asimismo, agradezco a Celeste Novelli, quien colaboró en la primera etapa de recolección de datos. Finalmente, agradezco a las/os evaluadores, cuyas observaciones potenciaron los resultados de esta investigación.

${ }^{\star *}$ Abogada (UBA). Doctoranda (UBA). Investigadora en formación en "Instituto de Investigaciones Jurídicas y Sociales A.L. Gioja", Facultad de Derecho, UBA. Docente de Derecho del Trabajo, UBA. Colaboradora OIT. Correo electrónico: jlobato@derecho.uba.ar. ORCID: http:// orcid.org/0000-0001-6881-5351 
hallazgos con los principales aportes del feminismo jurídico y los estándares vigentes en materia de derechos humanos de las mujeres.

Palabras clave: violencia contra las mujeres; derecho del trabajo; jurisprudencia; feminismos jurídicos.

\section{ABSTRACT}

This paper presents the analysis of the rulings of the Argentinian labor justice in cases of violence and workplace harassment against women. The main thesis argues that the labor judiciary reproduces the dynamics of sexist domination by not adapting the parameters of the judicial approach to the international standards regarding the protection of women's rights. For this, we will explore a sample of jurisprudence of the National Chamber of Labor Appeals in which we will address two main issues: the conceptions of violence against women at work and the configuration of the evidence and distribution of the probation burden. Then, we contrast these findings with the main contributions of legal feminism and the current standards in women's international human rights.

Keywords: Violence against women; labor law; jurisprudence; legal feminism.

\section{RESUMO}

Este trabalho explora os standards que emprega a jurisprudência em casos de violência laboral contra as mulheres. A tese principal sustenta que a justiça laboral reproduz dinâmicas de dominação sexista, ao não adequar os parâmetros de abordagem judicial aos standards internacionais vigentes em matéria de proteção dos direitos das mulheres. Para isso, analisaremos uma amostra de jurisprudência da Câmara Nacional de Apelações do Trabalho, na que atenderemos a duas questões principais: por um lado, às concepções de violência contra as mulheres nos âmbitos do trabalho e, por outro lado, à configuração do quadro provatório e a distribuição das cargas de prova. Depois, contrastaremos estes resultados com os principais aportes do feminismo jurídico e os standards vigentes em matéria de direitos humanos das mulheres.

Palavras-chave: violência contra as mulheres; direito do trabalho; jurisprudência; feminismos jurídicos. 


\section{Introducción}

La violencia contra las mujeres es un clivaje fundamental de la epistemología jurídica feminista. Los primeros abordajes se remontan a mediados de los años setenta del siglo $\mathrm{XX}$, cuando las feministas radicales norteamericanas identificaron la violencia sexual como fenómeno particular que afectaba a las mujeres y se ejercía con el objetivo de mantener dinámicas sociales de subordinación en razón del sexo (MacKinnon, 1979). A partir de estos pioneros estudios, la teoría jurídica feminista ha desentrañado el carácter estructural de la violencia hacia las mujeres, poniendo de relieve su anclaje en las desigualdades sociales producto del sistema sexo-género (Bodelón, 2006). Estos estudios se han potenciado a partir del desarrollo del Derecho Internacional de los Derechos Humanos de las Mujeres, identificando la violencia hacia las mujeres como una violación a los derechos humanos (Costa, 2016; Hein, 2016; Clérico \& Novelli, 2016; Bórquez, 2017). Esto implicó poner el acento sobre su carácter estructural: la violencia que padecen las mujeres tiene causas polisémicas y complejas que se vinculan con pautas culturales, económicas y sociales profundamente arraigadas en la sociedad. En efecto, la violencia contra las mujeres no puede ser concebida como hechos aislados, sino que es preciso entender el com-

plejo entramado de relaciones de poder asimétricas en el que se inserta (Hasanbegovic, 2017). Este giro supuso mutar los marcos explicativos desde nociones centradas en la culpabilidad individual - discursos muy ligados a retóricas patologizantes de los varones violentos-, a nociones sistémicas que conciben a la violencia como una forma extrema de discriminación (Barrére Unzueta, 2008). En lo que respecta al impacto de las desigualdades sexo-genéricas en el mundo del trabajo, algunos estudios han abordado esta cuestión desde los estudios organizacionales (Vázquez \& Urbiola, 2014; Neffa, 2015) y desde el derecho del trabajo (Casas Baamonde, 2019; Lobato, 2019a, 2019b; Pautassi et al., 2004).

En este panorama, cobra especial relevancia el accionar de las instancias jurisdiccionales tanto para garantizar el acceso a la justicia, como para brindar respuestas transformadoras que redefinan los criterios de justicia social. Las sentencias judiciales son dispositivos productores de narrativas sociales que solidifican las formas posibles de vida. Así, el 
derecho representa un territorio donde se inscriben las lecturas legitimadas socialmente. En ese sentido, se toma la definición de Bourdieu (2000), quien entiende al derecho como

la forma por excelencia del poder simbólico de nominación que crea las cosas nombradas y, en particular los grupos; el derecho confiere a esas realidades surgidas de sus operaciones de clasificación toda la permanencia, la de las cosas, que una institución histórica es capaz de conferir a instituciones históricas (p. 202).

De tal forma, tanto el derecho como los procesos judiciales son arenas de disputa que ofrecen una oportunidad para explorar las pedagogías judiciales que resultan disciplinantes hacia las mujeres.

Por otra parte, en el contexto latinoamericano todavía no existe una batería sólida de estudios científicos que analicen las implicancias de la violencia y acoso laboral (Ansoleaga et al., 2015). Estos estudios son escasos en la disciplina jurídica, donde solo destacan algunas producciones aisladas, que caracterizan al acoso y la violencia laboral como un proceso complejo de afectación a los derechos fundamentales que, además, se vincula con las modalidades de organización del trabajo y las estructuras empresariales (Morales Ramírez, 2016; Picón, 2009; Vázquez, 2009; Ugarte Cataldo, 2012). Así, el acoso laboral se manifiesta de diversas formas (violencia verbal, manipulación psicológica o aislamiento, entre otros) y puede producirse entre pares (horizontal), de personal jerárquico hacia sus dependientes (vertical-descendente) o de los dependientes hacia el personal jerárquico (vertical-ascendente). Asimismo, suele desarrollarse a través de periodos prolongados de tiempo, en el que se transitan diversas fases en las que, generalmente, las manifestaciones violentas suelen escalar (Caamaño Rojo \& Ugarte, 2014).

La escasa literatura existente confluye en señalar que el acoso y la violencia laboral afectan en mayor medida a las mujeres, quienes son las víctimas más frecuentes. ${ }^{1}$ En efecto, las violencias contra las mujeres, en los ámbitos de trabajo, muestran dinámicas segregacionistas que tienen

\footnotetext{
1 Se pueden consultar las estadísticas elaboradas por el Instituto Nacional de las Mujeres (https://bit.ly/2QzClGG) y por la Oficina de Asesoramiento sobre Violencia Laboral (https:// www.argentina.gob.ar/trabajo/oavl/estudios).
} 
su raíz en la subordinación histórica de las mujeres. Así, una de las claves principales es el desempeño de las mujeres en trabajos y roles distintos a los asignados tradicionalmente, desafiando normativas de género profundamente arraigadas en la sociedad. De esta forma, el énfasis se traslada a las lógicas desigualitarias de distribución del poder y a las relaciones asimétricas que se producen en los ámbitos laborales (Lobato, 2019c).

Con estas discusiones dialoga el presente trabajo. El interés es indagar sobre la forma en que la justicia laboral ordinaria en Argentina aborda los casos de violencia de género en los ámbitos laborales; el objetivo central es explorar los casos de violencia y acoso laboral que resolvió la Cámara Nacional de Apelaciones del Trabajo Argentina en los últimos años. Si bien este trabajo posee un fuerte cariz local, contribuye a generar diálogos más extensos para analizar el abordaje que los operadores jurídicos laborales y sociales hacen de la violencia contra las mujeres en los lugares de trabajo.

La tesis principal que se demostrará es que la justicia laboral de Argentina mantiene y reproduce dinámicas de dominación sexista, al no adecuar los parámetros de abordaje judicial a los estándares vigentes en materia de protección de los derechos de las mujeres. Para tal fin, en primer lugar se hará un relevamiento de la normativa nacional e internacional aplicable en la materia. Ello proporcionará el marco necesario para analizar la muestra de jurisprudencia en base a dos claves de lectura principales: las definiciones de violencia y la conformación del marco probatorio. En virtud de este análisis estableceremos algunos puntos de discusión centrales para avanzar hacia el robustecimiento de las categorías jurídicas aplicables a casos de violencia laboral contra las mujeres. El trabajo finaliza con algunas reflexiones que proyectan hilos de continuidad de la presente investigación.

\section{Marco normativo vigente en Argentina sobre violencia laboral contra las mujeres}

En Argentina, las mujeres configuran un grupo de especial tutela constitucional de acuerdo a la cláusula contenida en el artículo 75.23, que sostiene como una de las atribuciones del Congreso: 
Legislar y promover medidas de acción positiva que garanticen la igualdad real de oportunidades y de trato, y el pleno goce y ejercicio de los derechos reconocidos por esta Constitución y por los tratados internacionales vigentes sobre derechos humanos, en particular respecto de los niños, las mujeres, los ancianos y las personas con discapacidad.

De esta forma, la Constitución Nacional argentina (CNA) reconoce que las mujeres son un grupo que requiere de especial protección, por encontrarse en una situación de subordinación social (Saba, 2007; Lobato, 2019a).

En el marco del Derecho Internacional de los Derechos de las Mujeres, la adopción y ratificación por parte de Argentina de la Convención sobre la Eliminación de Todas las Formas de Discriminación contra la Mujer (CEDAW), en el ámbito universal, y de la Convención Interamericana para Prevenir, Sancionar y Erradicar la Violencia contra la Mujer (Convención Belém do Pará), en el ámbito interamericano, han sido hitos en el desarrollo del derecho de las mujeres a una vida libre de violencias. Esta situación se ha visto robustecida en Argentina por su elevación a jerarquía constitucional en la reforma de 1994 (artículo 75.22 CNA). Asimismo, en el ámbito de la OIT se adoptó, en 2019, el Convenio 190 sobre la eliminación de la violencia y el acoso en el mundo del trabajo y la Recomendación 206 (Lobato, 2019d), que se complementan con los convenios clásicos de igualdad de género de la OIT (1998) que, además, forman parte del decálogo de derechos fundamentales.

En lo que respecta a la legislación interna, la normativa troncal del derecho del trabajo en las relaciones privadas: la Ley de Contrato de Trabajo $^{2}$ (LCT), no prevé resortes protectorios específicos para situaciones

2 Ley 20.744 (t.o 1976). Algunos países de la región han abordado la materia mediante la sanción de leyes específicas para regular el acoso laboral, como Colombia (Ley 1010 del 2006); o introduciendo modificaciones a los Códigos del Trabajo, como los casos de Chile (Ley 20.607 de 2012), México (Ley Federal del Trabajo de 2012) y Venezuela (Ley Orgánica del Trabajo, de los Trabajadores y Trabajadoras de 2012). Otros países contienen cláusulas que prohíben el acoso en las Constituciones, como Bolivia (artículo 49.3: "El Estado protegerá la estabilidad laboral, prohibiéndose el despido injustificado y toda forma de acoso laboral") y Ecuador (artículo 66.3: “Se reconoce y garantizará a las personas (...) El derecho a la integridad personal, que incluye: a) La integridad física, psíquica, moral y sexual; b) Una vida libre de violencia en el ámbito público y privado" y el artículo 66.4: "Derecho a la igualdad formal, igualdad material y no discriminación"). 
de violencia laboral, sino solo normas que prohíben la discriminación (artículos 17 y 81 de la LCT) ${ }^{3}$ y deberes de conducta genéricos de las partes (artículos 62, 63 y 75 de la LCT). Esta circunstancia se vincula con que el modelo de relaciones laborales al que responde el ordenamiento jurídico laboral argentino es de tipo fordista clásico y no ha incorporado aún las teorías sobre protección robusta a los derechos fundamentales y ciudadanía en la empresa (Arese, 2014; Livellara, 2015; Rodríguez Mancini, 2007; Sen, 2000; Zas, 2014). Sin perjuicio de ello, diversas provincias han adoptado leyes que regulan el acoso laboral en el empleo público. ${ }^{5}$ Poco a poco ciertos convenios colectivos han comenzado a incorporar cláusulas relativas a violencia de género y violencia laboral. ${ }^{6}$

3 Artículo 17 de la LCT: "Por esta ley se prohíbe cualquier tipo de discriminación entre los trabajadores por motivo de sexo, raza, nacionalidad, religiosos, políticos, gremiales o de edad"; artículo 81 LCT: "El empleador debe dispensar a todos los trabajadores igual trato en identidad de situaciones. Se considerará que existe trato desigual cuando se produzcan discriminaciones arbitrarias fundadas en razones de sexo, religión o raza, pero no cuando el diferente tratamiento responda a principios de bien común, como el que se sustente en la mayor eficacia, laboriosidad o contracción a sus tareas por parte del trabajador".

4 Artículo 62 LCT: "Las partes están obligadas, activa y pasivamente, no sólo a lo que resulta expresamente de los términos del contrato, sino a todos aquellos comportamientos que sean consecuencia del mismo, resulten de esta ley, de los estatutos profesionales o convenciones colectivas de trabajo, apreciados con criterio de colaboración y solidaridad"; artículo 63 LCT: "Las partes están obligadas a obrar de buena fe, ajustando su conducta a lo que es propio de un buen empleador y de un buen trabajador, tanto al celebrar, ejecutar o extinguir el contrato o la relación de trabajo"; artículo 75 LCT: "El empleador debe hacer observar las pautas y limitaciones a la duración del trabajo establecidas en la ley y demás normas reglamentarias, y adoptar las medidas que según el tipo de trabajo, la experiencia y la técnica sean necesarias para tutelar la integridad psicofísica y la dignidad de los trabajadores, debiendo evitar los efectos perniciosos de las tareas penosas, riesgosas o determinantes de vejez o agotamiento prematuro, así como también los derivados de ambientes insalubres o ruidosos.

Está obligado a observar las disposiciones legales y reglamentarias pertinentes sobre higiene y seguridad en el trabajo. El trabajador podrá rehusar la prestación de trabajo, sin que ello le ocasione pérdida o disminución de la remuneración, si el mismo le fuera exigido en transgresión a tales condiciones, siempre que exista peligro inminente de daño o se hubiera configurado el incumplimiento de la obligación, mediante constitución en mora, o si habiendo el organismo competente declarado la insalubridad del lugar, el empleador no realizara los trabajos o proporcionara los elementos que dicha autoridad establezca".

5 Es el caso de la Ley 1.225 en el ámbito de la Ciudad Autónoma de Buenos Aires, la Ley 13.168 en la provincia de Buenos Aires, la Ley 5.349 en Jujuy, la Ley 7.232 en Tucumán, la Ley 12.434 en Santa Fe y la Ley 7.006 en Chaco.

6 Algunos ejemplos son el Convenio General para la Administración Pública Nacional (dec. 31/2018) en el ámbito público, el Convenio Colectivo de Trabajo de la Industria Indumentaria (nro. 501/07), el Convenio Colectivo de Trabajo de Trabajadores de YPF (nro. 1572/18 "E"). En relación a las centrales sindicales, la Central de Trabajadores de la Argentina (СTA-T) ha sido pionera en los avances en materia de género, elaborando diferentes diseños desde la "Secretaría de Género e Igualdad de Oportunidades". En ese sentido, en octubre 
Por otra parte, en el año 2009 se sancionó la Ley 26.485 (Ley de Protección integral para prevenir, sancionar y erradicar la violencia contra las mujeres en los ámbitos en que desarrollen sus relaciones interpersonales), reglamentada a través del Decreto 1011/2010. Esta norma define la violencia en los siguientes términos:

Se entiende por violencia contra las mujeres toda conducta, acción u omisión, que de manera directa o indirecta, tanto en el ámbito público como en el privado, basada en una relación desigual de poder, afecte su vida, libertad, dignidad, integridad física, psicológica, sexual, económica o patrimonial, como así también su seguridad personal. Quedan comprendidas las perpetradas desde el Estado o por sus agentes (artículo 4). ${ }^{7}$

Por su parte, el artículo 5 establece diferentes tipos de violencia entre las que se encuentran la física, psicológica, sexual, económica y patrimonial y simbólica. El artículo 6 (inciso c) establece diferentes modalidades de violencia entre las que se encuentra la laboral:

aquella que discrimina a las mujeres en los ámbitos de trabajo públicos o privados y que obstaculiza su acceso al empleo, contratación, ascenso, estabilidad o permanencia en el mismo, exigiendo requisitos sobre estado civil, maternidad, edad, apariencia física o la realización de test de embarazo. Constituye también violencia contra las mujeres en el ámbito laboral quebrantar el derecho de igual remuneración por igual tarea o función. Asimismo, incluye el hostigamiento psicológico

de 2017 ya había impulsado un plan de acción denominado "Compromiso sindical para la erradicación de la violencia de género". A principios del 2019, fue la única central en apoyar el Paro Internacional de Mujeres del 8 de marzo (decisión adoptada en la Mesa Ejecutiva Nacional del 06 de febrero 2019) y en marzo aprobó el plan de acción para implementar el "Protocolo marco de prevención y acción para las organizaciones sindicales en casos de discriminación, acoso y violencia por razones de género contra las mujeres y personas LGBT+ en el ámbito laboral", para ser implantado en las organizaciones sindicales que componen la central.

7 Respecto de este artículo, la norma reglamentaria establece: “Se entiende por relación desigual de poder, la que se configura por prácticas socioculturales históricas basadas en la idea de la inferioridad de las mujeres o la superioridad de los varones, o en conductas estereotipadas de hombres y mujeres, que limitan total o parcialmente el reconocimiento o goce de los derechos de éstas, en cualquier ámbito en que desarrollen sus relaciones interpersonales". 
en forma sistemática sobre una determinada trabajadora con el fin de lograr su exclusión laboral.

Ahora bien, esta norma es complementada por las disposiciones del decreto reglamentario 1011/2010, que estipulan:

Se considera discriminación en el ámbito laboral cualquier omisión, acción consumada o amenaza que tenga por fin o por resultado provocar distinción, exclusión o preferencia basada en los motivos mencionados en la ley que se reglamenta o en cualquier otro motivo que tenga por efecto anular o alterar la igualdad de oportunidades o de trato, empleo u ocupación de las mujeres. En el mismo sentido, se entiende discriminatoria la exigencia, tanto sea para acceder como para mantener un contrato de trabajo, de cualquier requisito inherente a la pertenencia de género.

(...) Se considera hostigamiento psicológico a toda acción, omisión o comportamiento destinado a provocar, directa o indirectamente, daño físico, psicológico o moral a una trabajadora, sea como amenaza o acción consumada, y que puede provenir tanto de niveles jerárquicos superiores, del mismo rango o inferiores.

(...) En los supuestos de denuncia de discriminación por razón de género, resultarán aplicables los principios generales receptados en materia de prueba en el Convenio OIT 111 "Convenio relativo a la discriminación en materia de empleo y ocupación" sobre discriminación (empleo y ocupación de 1958) y lo expuesto por la Comisión de Expertos en Aplicación de Convenios y Recomendaciones de la Organización Internacional del Trabajo, Estudio General sobre Igualdad en el empleo y la ocupación, $75^{\circ}$ reunión Ginebra 1988, así como lo señalado en el Informe Global de la $96^{\circ}$ reunión de la Conferencia Internacional del Trabajo, 2007, n. ${ }^{\circ} 198 .^{8}$

Con todo, aunque en Argentina está vigente una norma de protección robusta a la violencia contra las mujeres, el hecho de que el sistema

8 Negrillas fuera del original. 
de la LCT no contemple este tipo de casos presenta diversas dificultades prácticas a los tribunales.

\section{Metodología}

La muestra de jurisprudencia se compone de 140 sentencias de la Cámara Nacional de Apelaciones del Trabajo de Argentina (CNAT). La elección de la jurisdicción correspondió a que la segunda instancia es, en la gran mayoría de los casos, la que zanja la suerte de los juicios laborales en Argentina., debido a que como el procedimiento judicial laboral tiene carácter gratuito para la parte trabajadora, ${ }^{9}$ la mayoría de las sentencias de primera instancia suelen ser apeladas. Sin embargo, en escasas oportunidades las sentencias de segunda instancia son objeto de recurso extraordinario federal. Esto se vincula a cuestiones de costos procesales, ${ }^{10}$ pero también a la admisibilidad del recurso y a la naturaleza de derecho común de la mayoría de las cuestiones ventiladas en los juicios laborales (artículo 14, Ley 48).

El recorte temporal corresponde al periodo comprendido entre el primero de febrero de 2016 y el 31 de diciembre de 2018, por ser el periodo más reciente de jurisprudencia laboral. No obstante, es necesario resaltar que la justicia laboral no posee - ni el sistema judicial argentino, en general-, un sistema integrado de sentencias publicadas; por esta razón, acceder a las mismas fue una tarea ardua. Para la recopilación se utilizaron diversos motores de búsqueda: La Ley, El Dial, RubinzalCulzoni, Centro de Información Judicial y los boletines temáticos de jurisprudencia de la CNAT, Género y Justicia. Para todos los buscadores se utilizaron las mismas palabras clave: "violencia de género", "igualdad de género", "discriminación + mujer", "estereotipos", "estereotipante", "CEDAW", "Belém do Pará" y "ley 26485". Con la selección de los motores

9 Artículo 20 de la LCT: “El trabajador o sus derecho-habientes gozarán del beneficio de la gratuidad en los procedimientos judiciales o administrativos derivados de la aplicación de esta ley, estatutos profesionales o convenciones colectivas de trabajo".

10 Actualmente en Argentina, el monto del depósito establecido en el artículo 286 CPCCN asciende a la suma de $\$ 40000$ (Acordada CSJN 42/2018). 
de búsqueda y las palabras clave se buscó desarrollar una recopilación lo más amplia posible.

\section{Resultados}

Antes de adentrarnos a un análisis de corte cualitativo sobre los aspectos más relevantes de la jurisprudencia, resulta prioritario establecer ciertos rasgos generales de las sentencias en estudio. Así, un primer aspecto fundamental es que el $100 \%$ de los reclamos se producen una vez extinta la relación laboral (tabla 1). Es decir, las mujeres que sufren violencia en los ámbitos del trabajo son despedidas o se ponen en situación de despido indirecto (artículos 242 y 246 de la LCT) ${ }^{11}$ a causa del mismo. En relación con la admisibilidad de los reclamos, de la totalidad de la muestra solo un $50 \%$ hace lugar al reclamo, mientras que un 47,14\% desestima la demanda. E1 2,86\% restante corresponde a resoluciones de medidas cautelares que todavía no se han expedido sobre el fondo.

Tabla 1. Admisibilidad de los reclamos

\begin{tabular}{|l|c|c|}
\hline \multicolumn{1}{|c|}{ Decisión } & Cantidad & Porcentaje \\
\hline Favorable & 70 & $50 \%$ \\
\hline Rechazo & 66 & $47,14 \%$ \\
\hline Resolución pendiente & 4 & $2,86 \%$ \\
\hline Total & 140 & $100 \%$ \\
\hline
\end{tabular}

Además, es posible clasificar las sentencias según la normativa en que se fundan (tabla 2). Así, la mayor parte de los pronunciamientos

11 Artículo 242 LCT: “Una de las partes podrá hacer denuncia del contrato de trabajo en caso de inobservancia por parte de la otra de las obligaciones resultantes del mismo que configuren injuria y que, por su gravedad, no consienta la prosecución de la relación. La valoración deberá ser hecha prudencialmente por los jueces, teniendo en consideración el carácter de las relaciones que resulta de un contrato de trabajo, según lo dispuesto en la presente ley, y las modalidades y circunstancias personales en cada caso"; artículo 246 LCT: "Cuando el trabajador hiciese denuncia del contrato de trabajo fundado en justa causa, tendrá derecho a las indemnizaciones previstas en los artículos 232, 233 y $245^{\prime \prime}$. 
utiliza las prescripciones de la normativa laboral argentina ${ }^{12}$ antes que la normativa específica sobre protección a las mujeres. ${ }^{13}$ Las disposiciones del derecho laboral que se aplican tienen que ver con la responsabilidad del empleador por el daño sufrido por las trabajadoras en el ámbito laboral, en vinculación con el deber de indemnidad que pesa sobre aquel. De tal modo, las normas más citadas son el deber de seguridad del artículo 75 de la $\mathrm{LCT}^{14}$ y el artículo 4.1. de la Ley de Riesgos del Trabajo (24.557) ${ }^{15}$ que, en muchos casos, son complementadas con la responsabilidad objetiva de los artículos 1753 y siguientes del Código Civil y Comercial de la Nación Argentina (CCyCN). ${ }^{16}$

Tabla 2. Fundamento jurídico de las sentencias

\begin{tabular}{|l|c|c|}
\hline \multicolumn{1}{|c|}{ Normativa aplicada } & Cantidad & Porcentaje \\
\hline Normas de género & 18 & $12,86 \%$ \\
\hline Normas laborales & 122 & $87,14 \%$ \\
\hline Total & 140 & $100 \%$ \\
\hline
\end{tabular}

\section{Las concepciones de violencia contra las mujeres en los ámbitos laborales}

El primer eje de análisis se vincula con las concepciones sobre lo que es la violencia contra las mujeres en los ámbitos de trabajo. El hallazgo

12 Las normas de la LCT revisadas anteriormente.

13 Esto es, los tratados internacionales de derechos humanos con jerarquía constitucional y la Ley 26.485.

14 Ver nota 8.

15 Artículo 4.1 de la Ley 24.557: “Los empleadores y los trabajadores comprendidos en el ámbito de la LRT, así como las ART están obligados a adoptar las medidas legalmente previstas para prevenir eficazmente los riesgos del trabajo. A tal fin y sin perjuicio de otras actuaciones establecidas legalmente, dichas partes deberán asumir compromisos concretos de cumplir con las normas sobre higiene y seguridad en el trabajo. Estos compromisos podrán adoptarse en forma unilateral, formar parte de la negociación colectiva, o incluirse dentro del contrato entre la ART y el empleador".

16 Artículo 1753 CCyCN: "Responsabilidad del principal por el hecho del dependiente. El principal responde objetivamente por los daños que causen los que están bajo su dependencia, o las personas de las cuales se sirve para el cumplimiento de sus obligaciones, cuando el hecho dañoso acaece en ejercicio o con ocasión de las funciones encomendadas". 
más importante es que la mayor parte de las sentencias ${ }^{17}$ analiza los casos bajo el prisma del mobbing. Esta categoría es una construcción jurisprudencial que no se encuentra incorporada en la legislación argentina; proviene de las ciencias naturales y de la observación del comportamiento animal y, en la mayoría de los casos, los jueces laborales aplican esta definición en forma mecánica. ${ }^{18}$ Tal es así que las sentencias analizadas lo conciben como

una intimidación silenciosa; una situación en la que una persona o grupo de personas ejerce una violencia psicológica extrema, en forma sistemática y recurrente, durante un período prolongado, sobre otra persona o grupo de personas en el ámbito de trabajo, con la finalidad de destruir las redes de comunicación de la víctima y su reputación, perturbando el ejercicio normal de sus labores, hasta lograr que abandone el lugar de trabajo. ${ }^{19}$

La utilización del mobbing para caracterizar en forma genérica las situaciones de violencia en los ámbitos laborales sin reparar en los determinantes de género condujo a la jurisprudencia a elaborar una regla altamente rígida. Estas definiciones impactan en forma negativa no solo los juicios en cuestión -dado que la mayoría implica el rechazo de la acción, salvo que la actora haya consolidado un marco probatorio frondoso-, sino también los términos simbólicos: envía un mensaje de que solo serán justiciables aquellas situaciones de violencia extrema, a

17 Por ejemplo: "C.M.M c. Reyna Hotel SRL s/despido", Sala VIII, 03/08/2016; "B.V.C.G. c. Soluciones Agrícolas SA y otros s/despido", Sala II, 25/08/2016; "C.J.G. c/ AMX Argentina SA s/ despido", Sala II, 09/07/2016; “T. R. I. c/ Plata y Plata I.C.S.A. S/despido", Sala X, 25/10/2016; "R.M.M.F C/ HSBC BA SA s/despido", Sala V, 17/11/2016; "S.S.G. c/ Autoridad Regulatoria Nuclear (ARN) y otro s/ otros reclamos-mobbing", Sala X, 20/04/2017; "W.E.J C/ PCDA SA s/despido", Sala VI, 24/05/2017; "A.M.G c. Datco SA s/despido", Sala II, 13/09/2017.

18 Para mayor análisis sobre el mobbing se puede consultar Lobato, 2019c.

19 Negrilla fuera del orignal.

En similar sentido: "M. Z. A. c/ Technisys S.A s/despido", Sala X, 15/03/2017, p. 2; "M. R. P. c/ Casino Buenos Aires SA y otros s/despido", Sala IX, 31/07/2018; “O. V. L. c/ CS Salud Sociedad Anónima y otro s/despido", Sala X, 15/05/2018; "R. A. N. c/ Rophe SA y otro s/despido", Sala I, 18/04/2018; “A.A.M.V c/ Accenture Service Center SRL s/despido", Sala II, 27/03/2018; "B.G.E c/ Silkey SA s/despido", Sala X, 12/12/2017; "B.M.I c/ Sarmiento Suites SRL s/despido", Sala X, 03/03/201. 
las que la víctima esté expuesta de forma reiterada y prolongada, y que el maltrato tenga una finalidad excluyente.

Otro grupo de sentencias utiliza un documento de la OIT (2003) para definir la violencia. ${ }^{20}$ La utilización de este documento permite flexibilizar la tipología severa anterior. Estas concepciones resaltan que la violencia se manifiesta a través de diversas formas de maltrato, que se vinculan con relaciones asimétricas de poder con el objetivo de generar un daño a la persona que es objeto del maltrato. De tal forma, definen la violencia en el lugar de trabajo como "Toda acción, incidente o comportamiento que se aparta de lo razonable mediante el cual una persona es agredida, amenazada, humillada o lesionada por otra en el ejercicio de su actividad profesional o como consecuencia directa de la misma" (OIT, 2003, p. 4).

Sin perjuicio de ello, la mayoría de estas sentencias toman esta definición en forma lineal y no incluyen la variable de género en el análisis. Incluso, en algunas causas, los testimonios dan cuenta de una intencionalidad disciplinadora del maltrato, cuando los testigos sostienen que el agresor violentaba exclusivamente o en mayor medida a mujeres. Sin embargo, estos datos no son tenidos en cuenta por los jueces actuantes.

Por último, un grupo muy reducido de fallos utiliza las nociones del derecho internacional de las mujeres y la Ley 26.485. ${ }^{21}$ En estos casos, la situación de acoso laboral se encuadra en la definición de violencia laboral proporcionada por este cuerpo normativo para dar cuenta de que el género es el elemento principal de la situación de violencia laboral. En la mayoría de estas sentencias se suele equiparar el mobbing con la violencia psicológica prevista por el juego de los artículos 4, 5 y 6 de la Ley 26.485. Es decir, estas sentencias no propugnan el cumplimiento

20 Por ejemplo: "Q.P.M c. Biek SA s/despido”, Sala VIII, 06/06/2016; “A.V.Y.M vs. Embajada Paraguay s/ despido", Sala I, 27/06/2016; "Sch. E.T c/Asociación Comunidad Israelita Sefardi Argentina CISA y otros s/despido", Sala VII, 29/06/2016; "A.D.J. c/ Hipódromo Argentino de Palermo SA s/despido", Sala I, 25/11/2016; "H.Y.A. c/ Telecom Argentina SA s/despido", Sala I, 31/05/2017; “A.L.B c/ Asociación Escuelas Lincoln s/despido", Sala VIII, 25/08/2017.

21 Algunas de las sentencias que se insertan en esta línea son: "S.D.F c. Lancuer SA y otro s/despido", Sala VII, 24/02/2017; "C.R., S.R c/ COTO C.I.C.S.A y otros s/despido", Sala I, 03/12/2018; "D.S.A., C.R. C/ Massalin Particulares SA S/despido", Sala I, 28/09/2018; "R. B. M. c/ Casa de Cambio Maguitur SA s/despido", Sala VIII, 05/06/2018; “A. A. C. c/ AAGSAYO s/ despido", Sala V, 06/07/2018; "G.F.R c/ Autopistas del Sol SA s/juicio sumarísimo", Sala VII, 28/11/2018; “O. V. R. c/ Banco Galicia s/despido”, Sala IX, 27/02/2018. 
de los requisitos que el grupo anterior de sentencias requería. Dentro de estas, destaca una particular en la que las juezas avanzan en un desarrollo robusto de la protección de las mujeres. Allí se sostiene que la violencia de género en el ámbito laboral es producto de

la construcción cultural que asigna atributos y conductas a las personas según su pertenencia a un sexo biológico, en donde rige un orden jerárquico - que ha sido naturalizado por siglos- favorable a los varones, al tiempo que se consolida la idea de inferioridad de las mujeres como una cuestión biológicamente dada, y se generan así las justificaciones necesarias para la supervivencia de esta ideología (Sentencia “C.R., S.R c/ COTO C.I.C.S.A y otros s/despido", 2018, p. 5).

De tal forma, la sentencia en estudio encuadra la violencia como una forma de discriminación que responde a estructuras sociales desigualitarias. En efecto, las magistradas sostienen que si bien la violencia puede afectar a diversas personas, a las mujeres las afecta en forma predominante debido a "la existencia de relaciones de poder asimétricas que configuran jerarquías que favorecen a los varones". Esta caracterización conduce a las juezas a adoptar la perspectiva de género:

como método a favor del principio de igualdad real y en contra de la discriminación y subordinación de la mujer, de lo contrario, incurrir en la omisión de cuestionar la discriminación de la mujer, implica patentizar la reproducción de estereotipos que operan como refuerzo a la subordinación (Sentencia "C.R., S.R c/ COTO C.I.C.S.A y otros s/despido", 2018, p. 5).

Desde este prisma, las juezas encuadran el marco normativo en el derecho internacional de las mujeres y sostienen que es necesario cumplir con el deber de diligencia que pesa sobre los tres poderes del Estado, con especial énfasis en las obligaciones que emanan de los estándares del derecho internacional de los derechos humanos:

En definitiva, a la luz de las normas nacionales e internacionales reseñadas, se advierte que la definición normativa que contiene la Ley 26485 se ajusta a los arts. 1 de la CEDAW, 7 incs. a y b de la 
Convención Belém do Pará y al Convenio 111 de la O.I.T y que en conjunto tales instrumentos tienen como objetivo proteger y promover derechos irrenunciables, integrales y obligatorios tendientes a obtener la igualdad de trato, combatir la discriminación, eliminar la violencia y lograr un ambiente laboral sano y saludable, en otras palabras, la posibilidad de ejercer el derecho humano fundamental a trabajar digna y decentemente (Sentencia "C.R., S.R c/ COTO C.I.C.S.A y otros s/ despido", 2018, p. 9).

Con todo, este fallo y el de "Borda"22 son los únicos precedentes que desarrollan un análisis robusto del bagaje teórico y normativo con el que deben abordarse los casos de violencia y discriminación contra las mujeres en los ámbitos de trabajo.

\section{La cuestión probatoria}

En la sección anterior se vio que las sentencias que conforman la muestra definen la violencia contra las mujeres en los ámbitos de trabajo de tres formas diferentes: algunas se sirven de la noción de mobbing laboral, otras se ciñen a las prescripciones de la OIT y otras utilizan los insumos de la Ley 26.485. Dentro de estas últimas se identificó únicamente una sentencia que recepta los estándares y efectúa un abordaje integral de la violencia contra las mujeres en los ámbitos laborales. Con todo, falta analizar cómo determinan los jueces el encuadre probatorio para tener por acreditada o desechada la situación de violencia laboral. Para ello, se sostendrá la tesis de que la definición adoptada por el tribunal a la hora de juzgar casos de violencia contra las mujeres, impacta en forma directa la delimitación del cuadro probatorio y la distribución de las cargas.

Dos preguntas estructuran el análisis: ¿Cómo se distribuyen las cargas de prueba? ¿Bajo qué criterios son valoradas? En relación con la primera cuestión, los resultados mostraron que en los casos en que se adoptaron la noción de mobbing y la definición de la OIT, la carga

22 "Borda, Erica c/ Estado Nacional (Ministerio de Trabajo, Empleo y Seguridad Social de la Nación y otros s/ acción de amparo". Se trata del primer caso de litigio estratégico en materia de discriminación en el acceso al trabajo que resuelve la CNAT (Lobato, 2019b). 
probatoria se atribuye con exclusividad a las mujeres accionantes. Así, el cuadro probatorio se construye en base a dos supuestos: componente subjetivo perverso e intencional + situación repetitiva y sistemática. ${ }^{23}$ Esta fórmula es nítida en la argumentación de las sentencias:

No encuentro acreditada una conducta de la demandada tendiente a anular a la actora, ni algún tipo de violencia sistemática y prolongada sobre la persona de la trabajadora, ni tampoco un ambiente laboral apto para desencadenar el deterioro de la salud de la trabajadora (M.R.P. C. Casino Buenos Aires SA y otros s/despido", 2018). ${ }^{24}$

Era carga de la parte acreditar el presupuesto de su pretensión y en el marco de la vía legal escogida. Ello no implica someterla injustamente, ni en violación del principio in dubio pro operario y del orden público laboral. La decisión de demandar debe ser precedida por una evaluación técnica de los elementos con que se cuenta para acreditar los hechos respecto de los cuales existirá, presumiblemente, controversia. Afirmado un hecho relevante por la pretensora, pesa sobre ella la carga de probarlo, lo que no significa imponerle alguna actividad, sino el riesgo de que su pretensión sea desestimada, si el hecho no resulta, de alguna manera, acreditado. ${ }^{25}$

No encuentro motivación para apartarme de las reglas que rigen el onus probandi en el art. 377 Cód. Proc. Civ. y Com. de la Nación, amén de que la recurrente no explica porque se debió aplicar la

23 En palabras de una sentencia: "El acoso moral laboral es definido en la doctrina médica, sociológica y jurídica como una situación creada por una persona o grupo de personas, quienes ejercen una violencia psicológica extrema, de forma sistemática, durante un tiempo prolongado y sobre una persona en particular", "R.A.N c/ Rophe SA y otro s/despido", Sala I, 18/04/2018, p. 3. En el mismo sentido: "B. G. E. c/ Silkey SA s/despido", Sala X, 12/12/2017; "B.S.V. c/ Safari Sport SRL y otros s/despido", Sala I, 19/04/2017; “F. L. E. C. c/ BBVA Banco Frances SA y otro s/despido", Sala III, 27/04/2018; “K. G. A. c/ Cía. Argentina de Marketing Directo SA s/despido, Sala I, 28/03/2017; “M. D. A. c/ Winkl SA s/despido", Sala X, 22/12/2017; "W.E.J. c/ PCDA SA s/despido", Sala VI, 24/05/2017; "R. A. N. c/ Rophe SA y otro s/despido", Sala I, 18/04/2018.

24 En igual sentido, "M.M.L c. Mallinckrodt Medical Argentina Limited s/despido", Sala IX, 13/06/2018; "D.F.D.V c. Asociación Señoras de la Conferencia de San José s/despido", Sala V, 26/06/2018, entre otras.

25 "V.R.M. c/ Segar Seguridad SRL y otro s/accidente-acción civil", Sala VIII, 19/12/2016, p. 5; “B.A.M. c. San Up SA y otro s/despido, Sala II, 27/02/2018; “A.M.V. c. Accenture Service Center", Sala II, 27/03/2018, entre otras. 
teoría de las cargas dinámicas probatorias, es decir, que la contraria se encontraba en mejores condiciones de proporcionar prueba de que su parte no incurrió en malos tratos, lo que implicaría demostrar una conducta negativa, por lo tanto era la demandante la que tenía que demostrar el daño producido y opino que no lo ha logrado ("B.V.C.G. c. Soluciones Agrícolas SA y otros s/despido", 2016).

Sin embargo, estas decisiones no reparan en la imposibilidad de probar el componente subjetivo y perverso del agresor; requerir la constatación de esta situación implica una asunción de la violencia y discriminación de género como mero problema interpersonal entre dos personas determinadas. Es decir, la jurisprudencia que sostiene esta línea se posa en una noción individualista de la violencia, que no la concibe como discriminación de género, cuya tutela es responsabilidad del Estado. Aún más, requerir la prueba de la repetición y sistematicidad de los actos violentos implica someter a las personas a un trato cruel por tiempos prolongados con la esperanza de poder ser reclamados judicialmente.

Por otra parte, las sentencias que se apoyan en nociones más amplias de violencia y discriminación establecen la aplicación de la teoría de las cargas dinámicas de la prueba. En este sentido, la actora debe conformar un cuadro indiciario, pero será el empleador demandado quien deberá aportar las pruebas que resulten conducentes para admitir que no existió discriminación. Esta línea fue desarrollada por la Corte Suprema de Justicia de la Nación Argentina en los casos "Álvarez", "Pellicori" y "Sisnero" (Lobato, 2019a). De este modo, estas sentencias establecen:

Sobre la carga de la prueba a los fines de demostrar la discriminación alegada, vale recordar que, en virtud de la naturaleza de esta cuestión en controversia, rige con amplitud el principio de la prueba dinámica, en el sentido que basta que la actora proporcione algunos indicios serios de haber sufrido discriminación, para que se invierta el onus probandi y se desplace hacia la empleadora la carga de acreditar la legitimidad de su obrar. Así está establecido en la reglamentación del art. 6 inciso c de la Ley n. 26485 (...), por el Decreto n. ${ }^{\circ}$ 1011/2010 que determina aplicables a supuestos como el que se analiza, los principios generales receptados en materia de prueba en el Convenio 111 de la 
Organización Internacional del Trabajo (OIT) ("A.V., Y.M. vs. Embajada de la República del Paraguay s. despido", Sala I, 27/06/2016, p. 3). ${ }^{26}$

En relación con la segunda cuestión, en consonancia con las disposiciones del Código Procesal y la práctica jurídica de los Estados modernos, los jueces laborales adoptan el sistema de valoración libre sobre la sana crítica. De este modo, en todas las sentencias analizadas la prueba fundamental es la testimonial. La suerte de la mayoría de los reclamos se basa en la información que hubieren podido aportar los testigos. Para la acreditación del daño, los jueces se valen de los peritajes psicológicos y psiquiátricos efectuados, pero son escépticos; la prueba fundamental la aportan los testigos. En este punto también advertimos una clara influencia de la concepción de violencia, dado que en muchos de estos casos los jueces desestimaron los reclamos aun cuando en el expediente constaban peritajes que daban cuenta del daño producido a las víctimas. Esto debido a que la parte actora no habría probado el nexo causal:

No acreditado que los padecimientos psíquicos de la accionante tuvieran como origen un maltrato por parte de sus superiores jerárquicos o que hubiese sido víctima de mobbing o "acoso laboral", en definitiva lo que informa el informe pericial médico obrante a fs. 221/223, ha sido en base a comentarios de la propia actora, que lucen carentes de respaldo fáctico ("R.M.M.F. c/ HSBC BA SA s/despido", CNAT, 2016, p. 2). ${ }^{27}$

\section{Discusión}

Del análisis de jurisprudencia efectuado es posible concluir que existe un desacople de las resoluciones judiciales frente a los estándares vigentes en materia de violencia contra las mujeres en los ámbitos laborales en dos sentidos principales:

26 En similar sentido: "M.V.S c. AMX Argentina S.A s/despido", Sala I, 01/04/2016; “C.M.A c. Laboratorio Mi Flora SRL s/despido", Sala I, 08/04/2016; “C.J.M. c/ Gestiva SA y otro s/despido", Sala I, 07/06/2016; “G.M.F c. NT Servicios y Cargas SA s/despido", Sala VII, 15/07/2016; “G. N. M. L. vs. Avifra SRL y otro s. despido", Sala V, 21/11/2016; “S.M.E c/ Jardín del Pilar SA y otro s/accidente-acción civil”, Sala X, 30/11/2016.

27 En similar sentido: “B. M. c/ Telefónica de Argentina SA y otro s/ accidente-acción civil”, Sala II, 22/09/2017; “B. M. I. c/ Sarmiento Suites SRL s/despido”, Sala X, 03/03/2017, entre otras. 
- Falta de adecuación de las definiciones de violencia utilizadas a la normativa vigente. Esto implica dejar de lado nociones rígidas construidas pretorianamente (mobbing) y aplicar el marco legal y constitucional vigente. De acuerdo a los fallos analizados, la violencia se presenta como un problema "individual" que tiene su razón de ser en relaciones interpersonales disfuncionales o en perfiles psicológicos determinados. Sin embargo, esta visión no coincide con los estándares de derechos humanos imperantes que definen la violencia como una forma de discriminación que expresa una estructura social desigualitaria.

- Escasa aplicación de los estándares de valoración de prueba que emanan del Derecho Internacional de los Derechos Humanos. La evidencia analizada mostró que en el proceso laboral existen reglas de apreciación probatoria que, a priori, se presentan como neutrales pero que al ser aplicadas a los casos de violencia contra las mujeres producen efectos discriminatorios. Esto se corrobora cuando se descalifican los reclamos por contar con un testigo único, cuando los testigos son indirectos o cuando tienen juicio pendiente. Como se dijo anteriormente, las violencias contra las mujeres suelen desarrollarse en ámbitos privados, con lo cual es muy probable que la víctima no cuente con testigos directos que hayan estado presentes y puedan dar cuenta detallada de cómo se produjeron los hechos, especialmente en los reclamos laborales, donde los/as compañeros/as de la víctima pueden continuar vinculados/as laboralmente al agresor. Por esto es poco probable que accedan a declarar en un juicio en contra de este. En virtud de ello, los testigos suelen ser personas que ya han sido desvinculadas y que, muy probablemente, estén en juicio con el empleador por deficiencias registrales o en el pago indemnizatorio. Sin perjuicio de ello, la circunstancia de poseer juicio pendiente es también un motivo de descalificación de las declaraciones testimoniales.

\section{Conclusiones}

El objetivo central del artículo fue dar cuenta del abordaje que los tribunales del trabajo en Argentina hacen sobre casos de violencia laboral 
contra las mujeres. Para ello, se analizó una muestra de jurisprudencia sobre la que se establecieron rasgos generales y que escudriñó a través de dos claves de lectura específicas: las concepciones de violencia y la cuestión probatoria. Este estudio permitió establecer diálogos con la frondosa producción de los estudios de género sobre violencia y su impacto en el derecho internacional de los derechos humanos.

La conclusión central de este trabajo sostiene que, en gran medida, los tribunales laborales argentinos no cumplen los estándares internacionales y nacionales de protección para garantizar a las mujeres un ambiente laboral libre de violencias. Sin perjuicio de la mirada local, estos resultados se proyectan a escala regional, en tanto la mayoría de los países latinoamericanos no posee instrumentos jurídicos potentes para garantizar una tutela judicial efectiva a las mujeres víctimas de violencia laboral. Esto se ve agudizado por un escaso interés de la literatura jurídica por atender estas situaciones. De este modo, es necesario avanzar hacia sistemas sólidos de protección laboral para los casos de violación extrema a los derechos fundamentales en el trabajo como los casos de violencia laboral contra las mujeres.

Esta circunstancia adquiere incluso mayor relevancia en el contexto actual, dado que la adopción del Convenio 190 por parte de la OIT ha puesto sobre la mesa la necesidad de eliminar la violencia y acoso en el mundo del trabajo, especialmente en el caso de la violencia de género. En tal sentido, las respuestas judiciales son una instancia fundamental a considerar, en tanto revisten espacios legitimados para disputar complejas dinámicas sociales, pero también se presentan como escenarios aptos para construir imaginarios emancipatorios.

\section{Referencias}

Ansoleaga, E., Gómez-Rubio, C., \& Mauro, A. (2015). Violencia laboral en América Latina: una revisión de la evidencia científica. Vertex. Revista argentina de Psiquiatría, 26, 444-52.

Arese, C. (2014). Derechos humanos laborales. Rubinzal-Culzoni. 
Barrére Unzueta, M. A. (2008). Género, discriminación y violencia contra las mujeres. En P. Laurenzo, M. A. Maquedam, \& A. Rubio (Coords.), Género, violencia y derecho (pp. 13-31). Del Puerto.

Bodelón, E. (2006). Falsas seguridades, inciertas libertades: el debate sobre la violencia de género. En J. Calvo González (Coord.), Libertad y seguridad. La fragilidad de los derechos (pp. 17-38). Sociedad Española de Filosofía Jurídica y Política.

Bórquez, N. (2017). Hacia una igualdad transformadora en las producciones de la Corte y de la Comisión Interamericana de Derechos Humanos. Derechos sociales, mujeres y maquilas. Revista Electrónica. Instituto de Investigaciones Ambrosio L. Gioja, (19), 82-117.

Bourdieu, P. (2000). Poder, derecho y clases sociales. Descleé de Brouwer.

Casas Baamonde, M. E. (2019). La igualdad de género en el Estado constitucional. Revista de Derecho Social, (88), 13-54.

Caamaño Rojo, E., \& Ugarte Cataldo, J. L. (2014). El acoso laboral: tutela y prueba de la lesión de los derechos fundamentales. Revista lus et Praxis, 20(1), 67-90.

Costa, M. (2016). Feminismos jurídicos. Didot.

Clérico, L., \& Novelli, C. (2016). La inclusión de la cuestión social en la perspectiva de género: notas para re-escribir el caso "Campo Algodonero" sobre violencia de género. Revista de Ciencias Sociales, (67), 453-487.

Hasanbegovic, C. (2017). Entre el texto y la realidad. Los estándares internacionales de derechos humanos ison de utilidad para las mujeres? Revista Anales de la Facultad de Ciencias Jurídicas y Sociales, 14(47), 297-340.

Hein, D. (2016). Mujeres y acceso a la justicia. Didot.

Livellara, C. A. (2015). Derechos fundamentales inespecíficos del trabajador: su problemática y aplicación en Argentina. Gaceta Laboral, (21)1, 9-39.

Lobato, J. (2019a). Cláusula de igualdad en el ámbito laboral y perspectiva de género. Aportes desde el derecho del trabajo argentino a partir del caso Sisnero. Revista de la Facultad de Derecho, 46, 1-48. https://doi.org/10.22187/ rfd2019n46a9

Lobato, J. (2019b). Ampliación de la matriz de igualdad en los tribunales ordinarios. El caso "Erica Borda" y la justicia laboral. Derecho y Ciencias Sociales, (21), 214-240. https://doi.org/10.24215/18522971e214-240

Lobato, J. (2019c). Potencialidades y limitaciones del mobbing como categoría jurídica. La violencia contra las mujeres. Revista La Causa Laboral, 73(18). https://bit.ly/2WJe9XL 
Lobato, J. (2019d). El Convenio núm. 190 y la Recomendación núm. 206 de la oit. Un avance decisivo hacia el fortalecimiento de la protección de derechos fundamentales en el trabajo. Revista de Derecho Laboral Actualidad, (2), 27-43.

MacKinnon, C. (1979). Sexual harassment of working women. Yale University Press.

Morales Ramírez, M. A. (2016). Aproximación al acoso laboral desde la legislación comparada. Boletín Mexicano de Derecho Comparado, 49(147), 71-98.

Neffa, J. C. (2015). Los riesgos psicosociales en el trabajo: contribución a su estudio. CEIL-CONICET.

OIT (1998). Declaración de la OIT relativa a los principios y derechos fundamentales en el trabajo y su seguimiento. Conferencia Internacional del Trabajo.

OIT (2003). Repertorio de recomendaciones prácticas sobre la violencia en el lugar de trabajo en el sector de los servicios y medidas para combatirla. Oficina Internacional del Trabajo.

Pautassi, L., Faur, E., \& Gherardi, N. (2004). Legislación laboral en seis países latinoamericanos. Avances y omisiones para una mayor equidad. CEPAL. https:// repositorio.cepal.org/bitstream/handle/11362/5922/1/S045390_es.pdf

Picón, L. N. (2009). La equidad de género en el ámbito laboral. Desarrollo cultural y vías operativas para lograrla. Revista de Derecho Laboral, 1(2), 333-76.

Rodríguez Mancini, J. (2007). Derechos fundamentales y relaciones laborales. Astrea.

Saba, R. (2007). (Des)igualdad estructural. En M. Alegre y R. Gargarella (comp..), El derecho a la igualdad: aportes para un constitucionalismo igualitario (pp. 163-196). Buenos Aires: Lexis Nexis.

Sen, A. (2000). Trabajo y derechos. Revista Internacional del Trabajo, (199)2, 129-140.

Ugarte Cataldo, J. L. (2012). El acoso laboral: entre el Derecho y la Psicología. Revista de Derecho de la Pontificia Universidad Católica de Valparaíso, 39, 221-31.

Vázquez, G. A. (2009). Aspectos jurídicos del acoso sexual en el trabajo. Revista de Derecho Laboral, 1(1), 425-52.

Vázquez, A. E Urbiola, A. (2014). El género como una perspectiva para el análisis de las organizaciones. Iztapalapa, Revista de Ciencias Sociales y Humanidades, (77), 159-189.

Zas, O. (2014). El impacto del derecho internacional de los derechos humanos laborales en el ordenamiento jurídico argentino. Revista chilena de Derecho del Trabajo y de la Seguridad Social, 9(5), 193-239. 